\title{
Probing the origin of adaptive aromaticity in 16-valence-electron metallapentalenes
}

\author{
Dandan Chen ${ }^{[a, b]}$, Dariusz W. Szczepanik ${ }^{[b, c]}$, Jun Zhu*[a], and Miquel Solà*[b] \\ Dedication ((optional))
}

[a] D. Chen, Prof. J. Zhu

State Key Laboratory of Physical Chemistry of Solid Surfaces and Collaborative Innovation Center of Chemistry for Energy Materials (iChEM), Fujian Provincial Key Laboratory of Theoretical and Computational Chemistry and Department of Chemistry, College of Chemistry and Chemical Engineering Xiamen University

Xiamen 361005 (China)

E-mail: jun.zhu@xmu.edu.cn

[b] D. Chen, Dr. D. W. Szczepanik, Prof. M. Solà

Institute of Computational Chemistry and Catalysis and Department of Chemistry

University of Girona

C/ M. Aurèlia Capmany, 69, 17003 Girona, Catalonia, Spain

E-mail: miquel.sola@udg.edu

[c] Dr. D. W. Szczepanik

K. Guminski Department of Theoretical Chemistry, Faculty of Chemistry

Jagiellonian University

Gronostajowa, 2, 30-387 Kraków, Poland

Supporting information for this article is given via a link at the end of the document.((Please delete this text if not appropriate))

\begin{abstract}
Species with adaptive aromaticity are aromatic in the ground and lowest-lying triplet excited states and they have normally intermediate singlet-triplet gaps. Few examples of compounds with adaptive aromaticity are known to date, including 16-valence-electron (16e) metallapentalenes. A sweeping search could be conducted to discover new members of this group, but efficient designs with an explicit strategy would facilitate the quest for new members of this elusive family. Density functional theory calculations and aromaticity evaluations have been performed to reveal the nature of triplet-state aromaticity in $16 \mathrm{e}$ metallapentalenes. Our results show that coordination of strong $\sigma$ - or $\pi$-donor ligands helps achieving adaptive aromaticity of $16 \mathrm{e}$ metallapentalenes by means of a spin delocalization mechanism. These results have important implications for understanding the unusual properties of the organometallic adaptive aromatics, leading the way to efficient design of new compounds with tunable singlet-triplet gaps.
\end{abstract}

\section{Introduction}

Aromaticity is a fundamental concept in chemistry. ${ }^{[1]}$ The most quintessential aromatic molecule, benzene, was isolated by Michael Faraday in $1825 .{ }^{[2]}$ According to the known Hückel's and Baird's rules, ${ }^{[3]}$ planar monocyclic species with $4 n+2 \pi$-electrons are aromatic in the ground state $\left(\mathrm{S}_{0}\right)$ and antiaromatic in the lowest triplet state $\left(T_{1}\right)$, while those with $4 n$ m-electrons have reversed aromatic character in the two states. In 2008, Mandado and coworkers ${ }^{[4]}$ proposed to fuse Hückel's and Baird's rules in a new rule stating that aromatic annulenes are those having $2 n+1$ $\alpha$ and $\beta$ m-electrons, whereas those having $2 n \alpha$ and $\beta \pi$ electrons are antiaromatic. ${ }^{[5]}$

To date, the aromaticity concept has been extended to not only organic molecules but also organometallic and all-metal systems. ${ }^{[6]}$ As an intriguing research topic, aromaticity is sophisticated due to its multidimensional manifestations in reactivity, geometry, energetics, magnetic response, and electron delocalization properties. All of these properties have been used to define numerous indices of aromaticity. ${ }^{[7]}$ Most of aromaticity studies are focused on the ground-state, while the excited-state aromaticity has been getting more and more attention especially in the field of photochemistry as state-of-the-art methodologies have emerged to perform excited-state analyses both experimentally and computationally. ${ }^{[8]}$ Excited state (anti)aromaticity provides a manner to fine tune the singlet-triplet energy gap of compounds and this is an important factor in the design of new materials that act as molecular motors, ${ }^{\left[{ }^{[]}\right.}$high-spin organic molecules, ${ }^{[10]}$ photoluminescence materials, ${ }^{[11]}$ or photovoltaic cells. ${ }^{[12]}$

One way to design aromatic compounds with intermediate singlet-triplet energy gap is by making use of the adaptive aromaticity concept, which refers to the preservation of aromatic character after a change in the electronic state (normally, from singlet to triplet). ${ }^{[13]}$ Species with adaptive aromaticity do not follow Baird's rule. ${ }^{[3 b]}$ Organometallic species with transition-metal elements are likely to exhibit unconventional aromaticity such as Craig-type Möbius aromaticity ${ }^{[14]}$ by using the $d$ orbitals as components of conjugated arrays that form circuits with delocalized electrons. ${ }^{[15]}$ To date only metallapentalene and metallapyridinium with osmium and ruthenium centers were confirmed to be aromatic in both the lowest-lying singlet and triplet states. ${ }^{[13,16]}$

The determination of 8-center-8-electron Craig-type Möbius aromaticity in osmapentalenes and osmapentalynes is not as simple as it seems. ${ }^{[17]}$ The transition-metal $d$ orbitals participate in not only the Möbius but also the Hückel systems, for which two different electron-count rules are used.[18] The complexity of aromaticity in metallacycles makes unambiguous electroncounting a difficult task, at least not as simple as it is for annulenes ${ }^{[19]}$ Frontier orbitals in the ground-state and the excited- 
state are undoubtedly associated with the adaptive aromaticity, as $\Pi \rightarrow \sigma^{*}$ and $\sigma \rightarrow \pi^{*}$ adiabatic transitions were found in the solely reported cases. ${ }^{[13]}$ Although it has been found that strong electron acceptors could achieve aromatic benzene derivatives in the $T_{1}$ state ${ }^{[20]}$ no specific strategy has been proposed for the prediction or even design of organometallic species being aromatic regardless of the electronic state, which requires a thorough understanding of how the adaptive aromaticity is realized. Here we demonstrate that the formation of adaptive aromaticity can be rationalized via spin delocalization mechanism tuned by the donor-abilities of ligands, ${ }^{[21]}$ and, for the first time, an efficient strategy for achieving adaptive aromaticity in 16-valence-electron (16e) metallapentalenes is proposed.

\section{Results and Discussion}

Unexpected violation of adaptive aromaticity. To examine the possibility to induce adaptive aromaticity by more transition-metal elements, we started from $\mathrm{Re}^{+}$(Group 7) and $\mathrm{Ir}^{3+}$ (Group 9), which are isoelectronic to $\mathrm{Os}^{2+}$ (Group 8) in the 16e osmapentalene. Singlet-triplet energy gaps $\left(\Delta E_{S T}=E_{T}-E_{S}\right)$ for these compounds are given in Table $S 4 . \Delta E_{S T}$ for these species range from 9.8 to $45.1 \mathrm{kcal} / \mathrm{mol}$. Aromaticity of the $16 \mathrm{e}$ metallapentalene $\mathbf{M} . \mathbf{X}(\mathbf{M}=$ $\mathrm{Re}^{+} / \mathrm{Os}^{2+} / \mathrm{Ir}^{3+}, \mathbf{X}=\mathrm{F}^{-}, \mathrm{Cl}^{-}, \mathrm{Br}, \mathrm{I}^{-}$) with two phosphine ligands (Figure 1a) was evaluated using the NICS(1)zz index. ${ }^{[22]}$ All the twelve complexes are aromatic in the $\mathrm{S}_{0}$ state with negative NICS $(1)_{z z}$ values (Re.X, -28.6 -29.0 ppm; Os.X, -26.1 -26.4 ppm; Ir.X, $19.3 \sim-19.7 \mathrm{ppm})$. As the first reported species with adaptive aromaticity, ${ }^{[13 a]} \mathbf{O s . C l}$ is aromatic in the $\mathrm{T}_{1}$ state. One might assume the rest species to be also aromatic in the $T_{1}$ state due to the isoelectronic character. However, unexpected aromaticity loss is observed for Ir.F, Ir.Cl, and Ir.Br (Figure 1b) with minor NICS $(1)_{z z}$ values $(2.5 \sim 5.1 \mathrm{ppm})$ in the $\mathrm{T}_{1}$ state. The rest of the systems with negative NICS(1) zz values (Re.X, -30.7 -31.8 ppm; Os.X, -22.4 - -23.8 ppm; Ir.I, -17.2 ppm) in the $T_{1}$ state can be considered as aromatic as in the $\mathrm{S}_{0}$ state.

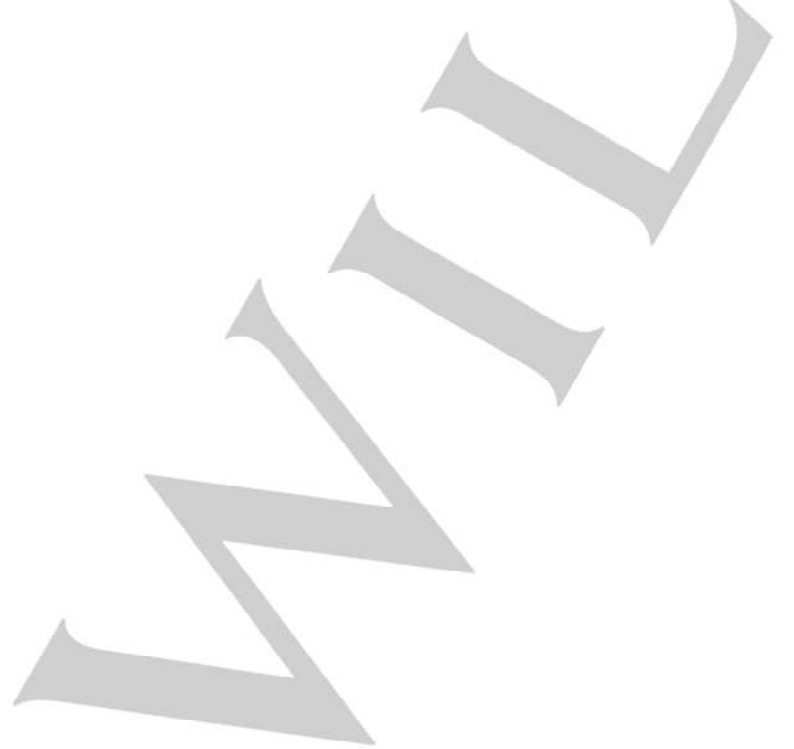

(a)

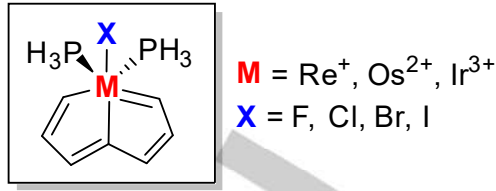

(b) $\operatorname{NICS}(1)_{z z}$ in the $S_{0} / T_{1}$ state

\begin{tabular}{|c|c|c|c|}
\hline Re.F & Re.CI & Re.Br & Re.I \\
\hline $29.0 /-31.8$ & $-28.6 /-31.1$ & $-28.6 /-30.9$ & $-28.6 /-30.7$ \\
\hline Os.F & Os.Cl & Os.Br & Os.I \\
\hline$-26.1 /-22.7$ & $-26.3 /-22.4$ & $-26.3 /-22.8$ & $-26.4 /-23.8$ \\
\hline Ir.F & Ir.CI & $\mathrm{Ir} \cdot \mathrm{Br}$ & Ir.I \\
\hline$-19.4 / 5.1$ & $-19.3 / 3.9$ & $-19.3 / 2.5$ & $-19.7 /-17.2$ \\
\hline
\end{tabular}

Figure 1. The geometry of 16 -valence-electron metallapentalene with one halide ligand and two phosphine ligands (a) and the NICS(1) zz values (unit: ppm) of M.X systems in the $S_{0}$ and $T_{1}$ states (b). Negative NICS $(1)_{z z}$ values result from diatropic ring currents in aromatic rings. Level of theory: (U)B3LYP/def2TZVP//(U)PBE0-D3BJ/6-31G(d) LanL2DZ.

Before raising a question of why only the iodide could make the iridapentalene aromatic in the $T_{1}$ state, the aromatic characters of $\mathbf{M} . \mathbf{X}$ should be examined using more approaches since aromaticity is a multidimensional phenomenon ${ }^{[7 c]}$. The anisotropy of induced ring current density $(A C I D)^{[23]}$ have been performed and the results (Figure S5) are in full agreement with $\mathrm{NICS}(1)_{z z}$ values, indicating nonaromaticity of Ir.F, Ir.Cl, and Ir.Br, and aromaticity of Ir.I in the $T_{1}$ state. It should be noted that the NICS $(1)_{z z}$ is not used as a quantitative index in this work because it could be influenced by the local magnetic response of metal centers. ${ }^{[24]}$ Particularly for Ir.Br and Ir.I, signed modulus density plots of GIMIC have been computed in comparison with typical aromatic (benzene) and antiaromatic (borole) molecules as well as a previously studied triplet-state antiaromatic osmacycle ${ }^{[13 a]}$ (Figure 2). Diatropic and paratropic GIMIC density surfaces above the cyclic $\sigma$ framework in benzene $\left(S_{0}\right)$ and borole $\left(S_{0}\right)$ are attributed to aromatic and antiaromatic $\pi$ systems, respectively (Figures 2a-2b). Aromatic characters of organometallic species are generally not as pronounced as in organic analogues. ${ }^{[6 b]}$ Paratropic GIMIC in the $\pi$ system of antiaromatic 18-valenceelectron osmapentalyne (Figure 2c) is weaker than that in borole but still highly recognizable. For $\operatorname{Ir} . \mathrm{Br}\left(\mathrm{T}_{1}\right)$, neither diatropic or paratropic $\pi$ density surfaces can be observed (Figure $2 d$ ). The Ir.I $\left(T_{1}\right)$ shows diatropic induced currents in the $\pi$ system (Figure $2 \mathrm{e}$ ), in line with its triplet-state aromaticity concluded from the NICS analysis. 
(a)

(b)<smiles>c1ccccc1</smiles>
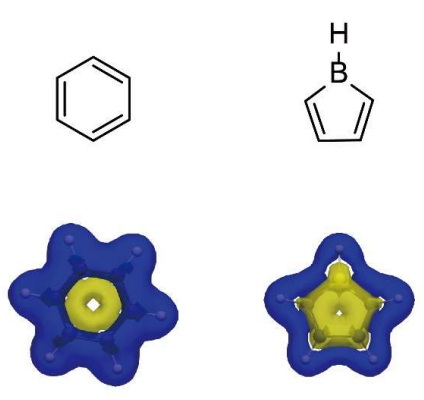

(c)
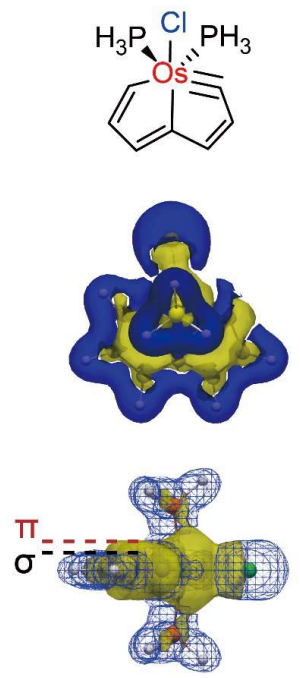

18e Osmapentalyne

$T_{1}$ (d)
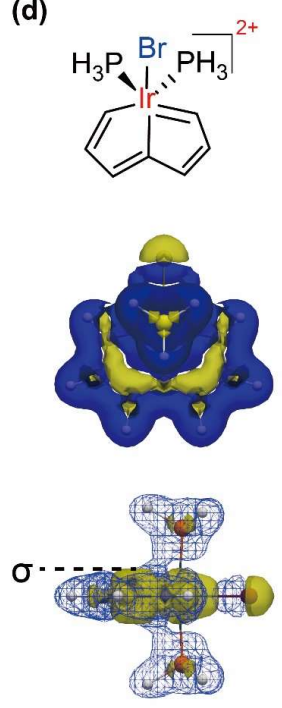

Ir.Br

$T_{1}$

non-aromatic (e)

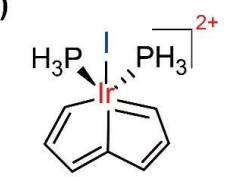

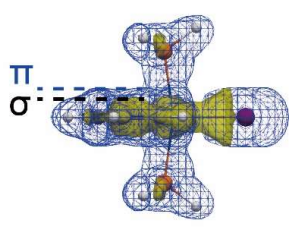

Ir.I

$\mathrm{T}_{1}$ aromatic anti-aromatic

aromatic

Figure 2. Signed modulus density plots of GIMIC (isovalue: 0.02) for characterizing $\pi$ aromatic characters of singlet-state benzene (a) and borole (b), and tripletstate osmacycles (c-e). Blue-solid and blue-wireframe density surfaces representing the same diatropic contributions are presented in top and side views, respectively. Yellow density surfaces correspond to paratropic contributions. Level of theory: (U)CAM-B3LYP/6-31G(d) LanL2DZ//(U)PBE0-D3BJ/631G(d) LanL2DZ.

Electron delocalization in the $\pi$ system of the eightmembered ring $(8 \mathrm{MR}$, including 7 carbon atoms and 1 metal atom) could be considerably different between Ir.Br and Ir.I in the $\mathrm{T}_{1}$ state to account for the different aromatic characters. $\pi$-EDDB analyses performed for the $8 \mathrm{MRs}$ in the $\mathrm{S}_{0}$ state indicate that the number of delocalized $\pi$-electrons in $\mathbf{R e} . \mathbf{X}(6.73 \sim 6.78 \mathrm{e}$, i.e. about 0.9 e per ring member, eprm - comparable to benzene $)^{[25]}$ is larger than in Os.X (5.11 5.40e, c.a. 0.7eprm - comparable to pyrrole $)^{[25]}$ and even larger than in Ir.X (4.00 4.02e, c.a. 0.5eprm - comparable to furan), ${ }^{[25]}$ which is coherent with the decreasing magnetic shielding ability $(\boldsymbol{R e} . X>0 s . X>I r . X)$ indicated by NICS results (Figure $1 b$ ). After the adiabatic transition from the $S_{0}$ to the $\mathrm{T}_{1}$ state, about $90 \%$ of delocalized $\pi$-electrons are retained in the 8MRs of Re.X. The $\pi$-EDDB of Os.X in the $T_{1}$ state is $94 \sim 99 \%$ of that in the $\mathrm{S}_{0}$ state, indicative of adaptive aromaticity. The Ir.I even has a slightly larger $\pi$-EDDB value in the $T_{1}$ state $(4.29 \mathrm{e})$ than in the $S_{0}$ state $(4.00 e)$. Dissection of $\pi-E D D B$ in the $T_{1}$ state shows that the $\pi$-electron delocalization in $\alpha$ and $\beta$ systems are comparable (Figure 3a) for species with adaptive aromaticity, indicating a well-preserved aromatic system against the change of electronic state. For Ir.F, Ir. $\mathbf{C l}$, and Ir.Br, the m-electron delocalization in the $\beta$ system is reduced by half in the $T_{1}$ state, while the overall $\pi-E D D B$ is only about $85 \%$ of that in the $S_{0}$ state. Isosurfaces of $\pi$-EDDB (Figure $3 b$ ) demonstrate the reduction of $\beta$ delocalization in $\operatorname{Ir} . \mathbf{B r}\left(T_{1}\right)$ and the adaptive aromaticity of Ir.I in the $\mathrm{S}_{0}$ and $\mathrm{T}_{1}$ states.

It is worth noting that the total m-electron population is the sum of the $\pi$-EDDB population (delocalized m-electrons), $\pi$-EDLA population ( $\pi$-electrons localized in atoms), and $\pi-E D L B$ population ( $\pi$-electrons localized between atomic pairs). ${ }^{[26]} \mathrm{We}$ end up with formally $6 \alpha$ m-electrons and $6 \beta$ m-electrons in the hybrid Hückel-Möbius aromatic 8MR fragment of Ir.I ( $\left.T_{1}\right)$, which clearly violates Mandado's rule predicting antiaromaticity for both $\alpha$ and $\beta$ subsystems (Figure S11). It has to be mention here that the fact that these metallacycles have a hybrid Hückel-Möbius character complicates electron counting and common rule following. ${ }^{[19 \mathrm{~b}]}$ Moreover, $6 \alpha$ m-electrons and $5 \beta$ m-electrons were found for all the rest $\mathbf{M} . \mathbf{X}\left(\mathrm{T}_{1}\right)$, even though Ir.F, Ir. $\mathbf{C l}$, and $\mathbf{I r} . \mathbf{B r}$ are nonaromatic while $\mathbf{R e} . \mathbf{X}$ and $\mathbf{O s . X}$ are aromatic. Hereby we conclude that the total $\pi$-electron population, and equivalently, the formal electron-count, are unable to rationalize the aromatic characters of M.X. Instead, the population of delocalized $\pi-$ electrons indicated by the $\pi$-EDDB is more relevant to the aromaticity especially for hybrid aromatic systems such as M.X. This conclusion is in full compliance with the recent findings that the strict correspondence between the formal number of $\pi-$ electrons and aromaticity does not hold for metallacycles with unbalanced $\Pi$-electron count (i.e. those $\mathbf{M}$.X having significantly more electrons than ring members). ${ }^{[19 b]}$

(a)

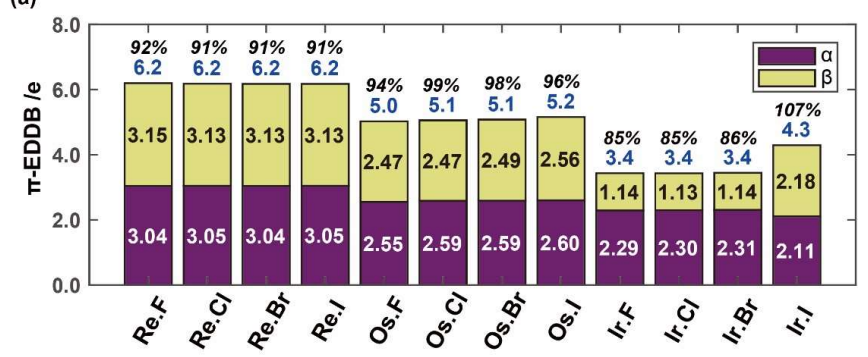

(b)

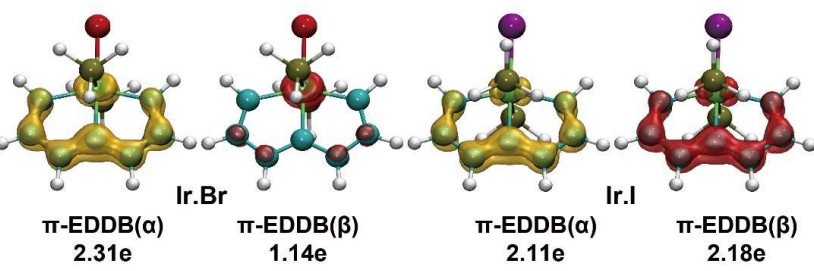

Figure 3. $\alpha / \beta$ dissection of $\pi-E D D B$ for the $8 M R$ of $\mathbf{M} . \mathbf{X}$ in the $T_{1}$ state. (a) Populations and (b) isodensity surfaces. Isodensity value for density surfaces: 
0.01 . Percentages are referred to $\pi-E D D B$ population in the $S_{0}$ state. Level of theory: (U)CAM-B3LYP/def2-TZVP//(U)PBE0-D3BJ/6-31G(d) LanL2DZ.

In the $T_{1}$ state, unpaired electrons belong to two singlyoccupied molecular orbitals (SOMOs) which in principle correspond to the HOMO and LUMO in the $S_{0}$ state as long as the balanced geometries are not drastically changed. Therefore, analyzing the frontier orbitals is one of the keys to understand the triplet-state aromaticity. Biorthogonalized SOMOs of Ir.X $\left(\mathrm{T}_{1}\right)$ are shown in Figure 4, all corresponding to the same SOMO sets of Re.X and Os.X (Figure S6). It has been shown that the highest SOMO (HSOMO) of 16e osmapentalene (Os.Cl) has no impact on the $\pi$-aromaticity due to its $\sigma$ character, while the HSOMO-1 is a $\pi$-bonding orbital with respect to the $8 \mathrm{MR}^{[13 a]}$ For the HSOMO-1 of Ir.I, the atomic orbital contribution from the iodine $(64.4 \%)$ is much higher than the $8 \mathrm{MR}(27.2 \%)$, in stark contrast to those of Ir.F, Ir.Cl, and Ir.Br where 8MRs have contributions larger than $90 \%$. Hence, the adaptive aromaticity of Re.X, Os.X, and Ir.I could be considered as an outcome of a transition involving negligible $\pi$-electrons from the 8MR. Meanwhile, considerable amounts of spin density with the out-of-plane $\pi$ character are distributed over the ring carbon atoms in Ir.F, Ir.Cl, and Ir.Br, whereas in Ir.I only two ligating carbon atoms share some spin density with $\sigma$ character (Figure 4).

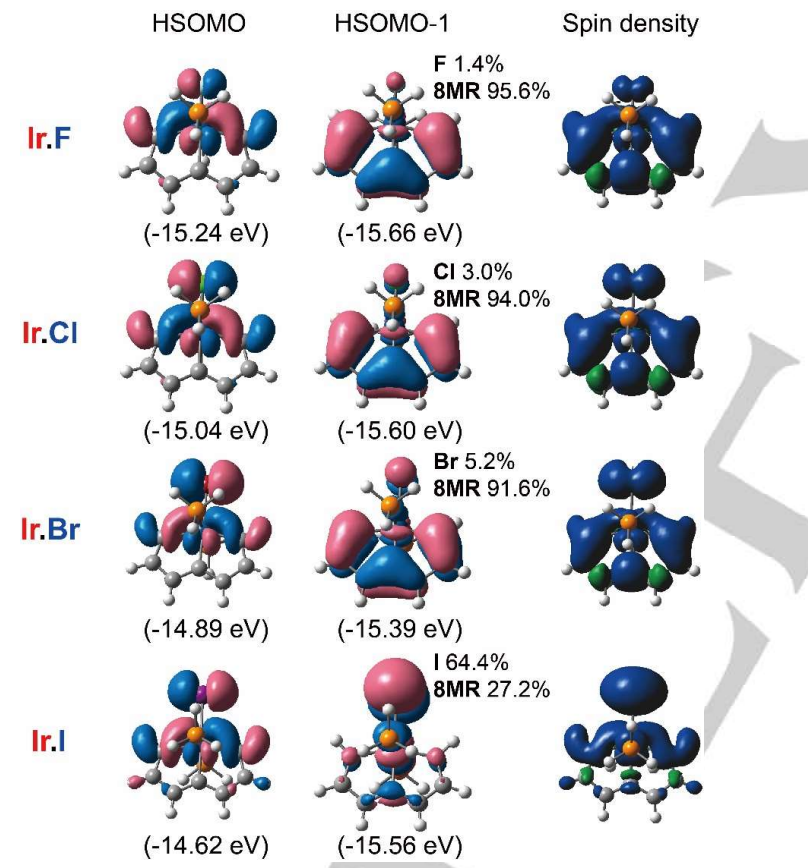

Figure 4. Biorthogonalized SOMOs and spin density distributions of $\mathbf{I r} . \mathbf{X}\left(\mathrm{T}_{1}\right)$. Isovalues for $\mathrm{MO}$ and spin-density contours are 0.03 and 0.001 a.u. respectively. Orbital compositions of halogen atoms and 8MRs (without hydrogen atoms) for the HSOMO-1 are provided.

The role of spin delocalization. The unexpected violation of adaptive aromaticity found in Ir.F, Ir. $\mathbf{C l}$, and Ir. $\mathbf{B r}$ has been rationalized by loss of $\pi$-electrons in the $8 \mathrm{MRs}$. However, the mechanism of ligand or metal-center effect in achieving adaptive aromaticity needs a more thorough explanation. Without the presence of $\mathbf{X}$ ligand, the metallacycles with frozen geometries are still aromatic in the $\mathrm{S}_{0}$ state, but after the vertical transition the $\left[\mathrm{Os}\left(\mathrm{PH}_{3}\right)_{2} \mathrm{C}_{7} \mathrm{H}_{6}\right]^{2+}$ and $\left[\mathrm{Ir}\left(\mathrm{PH}_{3}\right)_{2} \mathrm{C}_{7} \mathrm{H}_{6}\right]^{3+}$ are not aromatic in the unrelaxed $\mathrm{T}_{1}$ state while the aromaticity of $\left[\mathrm{Re}\left(\mathrm{PH}_{3}\right)_{2} \mathrm{C}_{7} \mathrm{H}_{6}\right]^{+}$is also reduced (Figure S8). Thus, the $\mathbf{X}$ ligand plays an important role in realizing the triplet-state aromaticity of $\mathbf{M} . \mathbf{X}$. Atomic spin populations in $\mathbf{M} . \mathbf{X}\left(\mathrm{T}_{1}\right)$ indicate that unpaired electrons are mainly distributed over the metal center and the ligating atoms after the adiabatic transition (Figure 5). Phosphine ligands only share negligible amounts of spin density (smaller than 0.05 ). For a given metal-center, the magnitude of spin-density shared by halide increases in the order of $\mathrm{F}^{-}<\mathrm{Cl}^{-}<\mathrm{Br}^{-}<\mathrm{I}^{-}$. The Re.X species exhibit highly localized spin density on the rhenium atom (population: 1.659 1.692 e), with noticeable distributions over no ring carbon atoms but halide ligands. Spin populations of halide ligands in Os.X are larger than those in $\mathbf{R e . X}$, while the varying ability of halides to share spin population is also more apparent in Os.X. In contrast, only $\mathrm{l}$ has a significantly larger spin population in Ir.X. The $\mathrm{F}^{-}, \mathrm{Cl}^{-}$, and $\mathrm{Br}^{-}$in $\mathbf{~ I r . X}$ are unable to share more spin density, whereas the spin populations of ligating carbon atoms are drastically increased to $0.336 \sim 0.468 \mathrm{e}$.

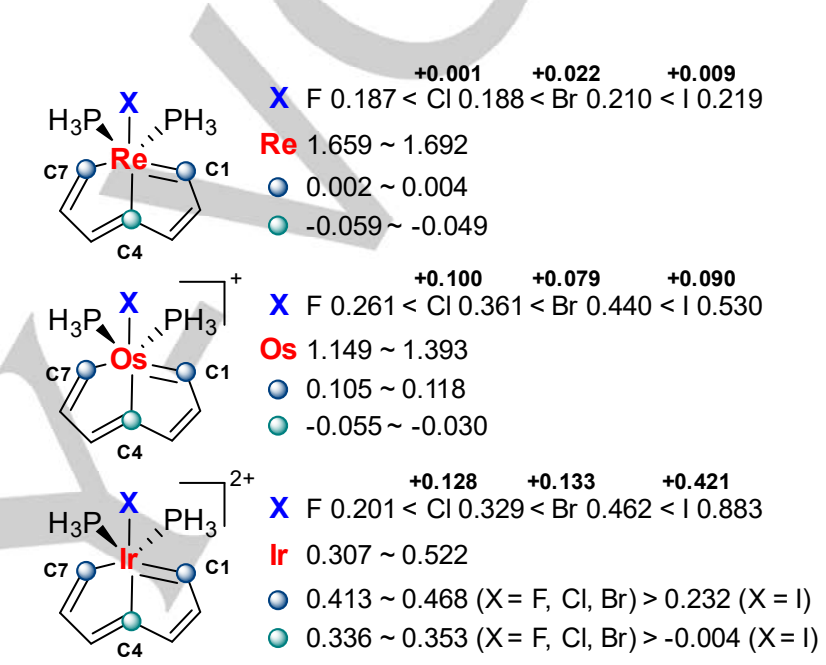

Figure 5. Atomic Mulliken spin populations (in e) in 16-valence-electron metallapentalenes. Positive and negative signs of spin population associate with $\alpha$ and $\beta$ spins, respectively. Spin populations of $C 1$ and $C 7$ are related to both HSOMO $(\sigma)$ and HSOMO-1 $(\pi)$, while the spin population of $\mathrm{C} 4$ is mainly determined by HSOMO-1 ( $\pi)$.

Spin delocalization and spin polarization are two mechanisms that decide how spin-density is distributed in transition metal complexes. ${ }^{[21]}$ Spin populations of the noncoordinated carbon atoms are minor $( \pm 0.01)$ in $\mathbf{M} . \mathbf{X}$, indicating minor spin polarization. Hence, spin delocalization is dominant in the triplet-state M.X. Instead of analyzing frontier orbitals, the formation of triplet-state metallapentalenes can be treated as a process where two unpaired electrons of the metal-center are partially delocalized toward the ligands. Such a delocalization is closely related with donor-ability of ligands ${ }^{[21]}$ Halogens are wellknown $\pi$-donor ligands in organometallic chemistry, with the ability increases in the order of $\mathrm{F}<\mathrm{Cl}<\mathrm{Br}<I^{\left[{ }^{[27]}\right.}$ This is consistent with what we have observed for the spin populations. The charge decomposition analysis (CDA) ${ }^{[28]}$ considering as fragments $\mathbf{M}$. and $\mathbf{X}$. (the fragments that results from homolytic dissociation of the $\mathbf{M}-\mathbf{X}$ bond) shows that the higher $\pi$-donating ability of halogen (characterized by higher energies of its valence $n p$ orbitals) leads to the larger contribution of its valence $n p$ orbitals in the HOMO, which has $\mathbf{M}-\mathbf{X}$ antibonding character (Figure 6 ). As a relatively weaker $\pi$-donor, fluorine has no considerable contribution to the HOMO of Ir.F. Both the $3 p_{z}$ spin orbitals of chlorine atom in Ir.Cl have $13 \%$ contribution to the corresponding spin HOMOs. The $4 p_{z}$ spin orbitals of bromine atom have higher contributions $(45 \%$, $51 \%)$ in Ir.Br. Even higher contributions from iodine (78\%, $77 \%$ ) 
are found in Ir.I where both $5 p_{z}$ and $5 p_{y}$ orbitals significantly interact with the metal center. Such an exceptional $\pi$-donor ability of iodine is prominent in the iridapentalene complex since the electron-deficiency of iridium center is higher than those of osmium and rhenium centers. Contribution of the $\mathrm{C}_{6} \mathrm{H}_{7}$ fragment to the HOMO orbital decreases in the order of increasing $\pi$-donor character, i.e., the largest contribution corresponds to $\mathbf{X}=\mathrm{F}$ and the smallest to $\mathbf{X}=\mathbf{I}$.

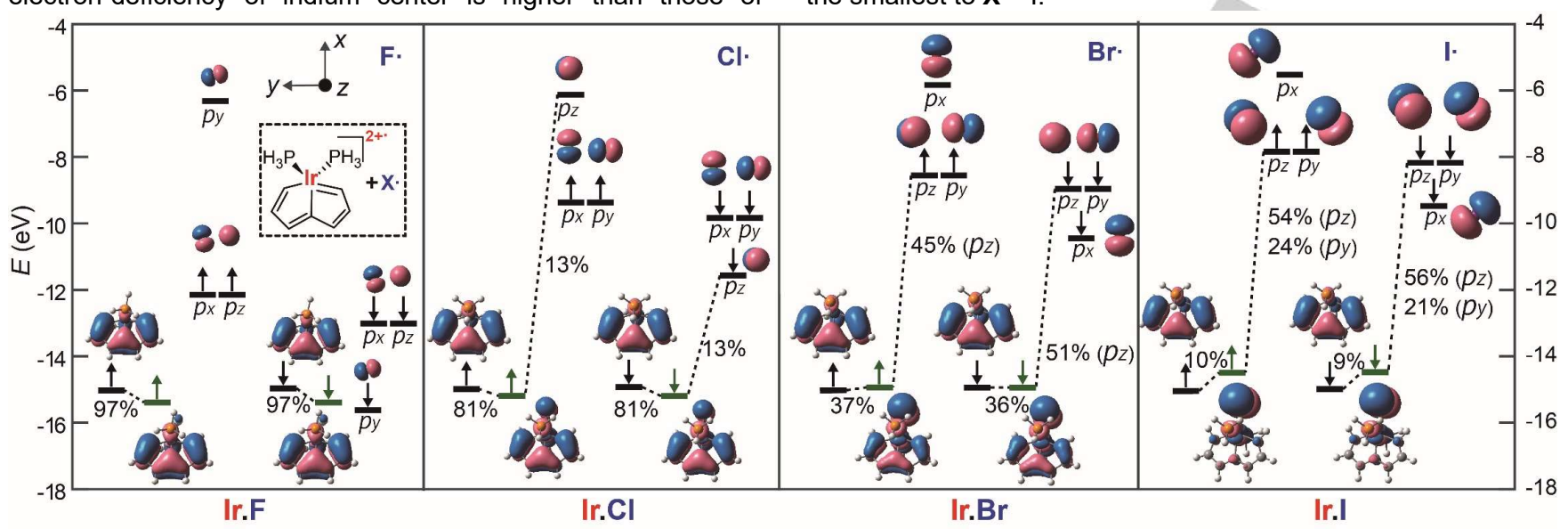

Figure 6. Orbital contributions obtained from charge decomposition analysis (CDA) for HOMOs of Ir.X in the So state. Each complex is divided into radical $\left[\mathrm{Ir}\left(\mathrm{PH}_{3}\right)_{2} \mathrm{C}_{7} \mathrm{H}_{6}\right]^{2+}$. and $\mathbf{X} \cdot(\mathrm{F} \cdot, \mathrm{Cl} \cdot$, Br-, or $\mathrm{I} \cdot)$. Smaller contributions from other orbitals are neglected. The spin flip was applied to $\mathbf{X} \cdot$, and thus its $\beta$ electrons outnumber the $\alpha$ counterparts. Upward and downward arrows represent $\alpha$ and $\beta$ electrons, respectively. Isovalue for orbital contours: 0.03 a.u. The LanL2DZ basis set is applied to all the halogens including the fluorine.

Strategy for achieving adaptive aromaticity. To test our hypothesis that strong donor ligands could be used to prevent spin delocalization from the metal-center to the $\mathrm{C}_{7} \mathrm{H}_{6}$ moiety and achieve adaptive aromaticity, we proceed to investigate a series of ligands with varying donor/acceptor ability for $16 \mathrm{e}$ metallapentalenes. The $\mathrm{PH}_{3}$ is used as a spectator ligand due to three reasons: 1) the spin delocalization from the metal center to Strong $\sigma$ donor Strong / Medium $\pi$ donor

\begin{tabular}{|c|c|c|c|c|c|c|}
\hline & $\mathrm{SnCl}_{3}^{-}$ & $\mathrm{BMe}_{2}^{-}$ & $\mathrm{NH}_{2}^{-}$ & $\mathrm{I}^{-}$ & $\mathrm{OH}^{-}$ & $\mathrm{Br}^{-}$ \\
\hline $\operatorname{Re}^{+}$ & $\begin{array}{c}-30.8 \\
0.095^{*}\end{array}$ & $\begin{array}{c}-29.7 \\
0.181^{*}\end{array}$ & $\begin{array}{c}\mathbf{- 3 2 . 4} \\
-0.054\end{array}$ & $\begin{array}{c}-30.7 \\
-0.049\end{array}$ & $\begin{array}{r}-32.3 \\
-0.057\end{array}$ & $\begin{array}{r}-30.9 \\
-0.051\end{array}$ \\
\hline$O s^{2+}$ & $\begin{array}{c}-26.1 \\
0.223^{*}\end{array}$ & $\begin{array}{c}-25.7 \\
0.285^{*}\end{array}$ & $\begin{array}{c}-27.1 \\
-0.052\end{array}$ & $\begin{array}{c}-23.8 \\
-0.030\end{array}$ & $\begin{array}{c}-25.4 \\
-0.054\end{array}$ & $\begin{array}{c}-22.8 \\
-0.033\end{array}$ \\
\hline $\mathrm{Ir}^{3+}$ & $\begin{array}{c}-18.7 \\
0.256^{*}\end{array}$ & $\begin{array}{c}-19.2 \\
0.353^{*}\end{array}$ & $\begin{array}{c}-20.1 \\
-0.045\end{array}$ & $\begin{array}{c}-17.2 \\
-0.004\end{array}$ & $\begin{array}{c}3.5 \\
0.345\end{array}$ & $\begin{array}{c}2.5 \\
0.336\end{array}$ \\
\hline
\end{tabular}

Spin population of C4 (a.u.) I the $\mathrm{PH}_{3}$ ligands is negligible; 2) the bulkiness of $\mathrm{PH}_{3}$ is small enough to minimize the steric effect; 3 ) $\mathrm{PH}_{3}$ is a commonly-used substitute of $\mathrm{PPh}_{3}$ in computational studies. ${ }^{[29]}$ The adaptive aromaticity of $\mathbf{M} . \mathbf{L}\left(\mathbf{M}=\mathrm{Re}^{+} / \mathrm{Os}^{2+} / \mathrm{Ir}^{3+}, \mathbf{X}=\mathrm{NH}_{2}{ }^{-}, \mathrm{OH}^{-}, \mathrm{F}^{-}, \mathrm{Cl}^{-}, \mathrm{Br}, \mathrm{I}^{-}\right.$, $\mathrm{H}_{2} \mathrm{O}, \mathrm{NH}_{3}, \mathrm{PMe}_{3}, \mathrm{CO}, \mathrm{PF}_{3}, \mathrm{H}^{-}, \mathrm{SnCl}_{3}{ }^{-}, \mathrm{BMe}_{2}^{-}$) has been evaluated (Figure 7)

Adaptive aromaticity in

Medium $\sigma$ donor / Strong $\pi$ acceptor

Hydride

\section{एyse}

Figure 7. Ligand effect on the triplet-state aromaticity of 16-valence-electron metallapentalenes. The spin population of C4 is indicative of the $\pi$-spin delocalization except for $\mathbf{M . S n C l}_{3}$ and $\mathbf{M} . \mathrm{BMe}_{2}$ whose SOMOs are all $\sigma$ orbitals. Spin population values marked with asterisks are attributed to $\sigma$-spin density only. All species have positive singlet-triplet energy gaps $\left(E_{T}-E_{S}\right)$, and they are aromatic in the $S_{0}$ state except for Ir.H $\left(S_{0}\right)$ which has a broken structure (Tables $S 3$ and $\left.S 4\right)$.

With a relatively electron-rich rhenium center, the Re.L has adaptive aromaticity even attached with a strong $\pi$-acceptor ligand such as $\mathrm{CO}$ or $\mathrm{PF}_{3}$. The Os.L displays adaptive aromaticity with strong m-donor $\left(\mathrm{NH}_{2}^{-}, \mathrm{OH}^{-}, \mathrm{F}^{-}, \mathrm{Cl}^{-}, \mathrm{Br}^{-}, \mathrm{I}^{-}\right)$or $\sigma$-donor ligands $\left(\mathrm{SnCl}_{3}{ }^{-}, \mathrm{BMe}_{2}{ }^{-}\right)$. For the Ir.L, where the iridium center is highly electron-deficient, it requires stronger donor ligands $\left(\mathrm{NH}_{2}{ }^{-}, \mathrm{I}^{-}\right.$, $\mathrm{SnCl}_{3}{ }^{-}, \mathrm{BMe}_{2}{ }^{-}$) to be aromatic in the $\mathrm{T}_{1}$ state. It should be noted that, to generate adaptive species, placing the $\mathbf{L}$ at the equatorial position as in the above-mentioned M.L systems is a better choice than at the axial positions. Interaction between $\mathbf{L}$ and the 8MR could be strong enough to cause drastic changes of chemical bonding if $\mathbf{L}$ is placed at the axial position (Figure $\mathbf{S 7}$ ).
Although both $\sigma$ - and $\pi$-donor ligands can promote the formation of adaptive aromaticity, the mechanisms are not exactly the same. $\pi$-donor ligand promotes adaptive aromaticity by facilitating the spin delocalization from metal-center to the donor ligand instead of the 8MR. This mechanism applies to systems with a $\pi-S O M O$ such as the HSOMO-1 of Ir.X (Figure 4). With a particularly strong $\pi$-donor such as $\mathrm{NH}_{2}^{-}$, adaptive aromaticity can be achieved even in the iridapentalene complex (Figure 8a). On the other hand, $\sigma$-donor ligands lead to two $\sigma$-SOMOs (Figure $8 b$ ) with no influence on the $\pi$-aromaticity. However, strong $\sigma$-donors could cause deformed geometries in the $S_{0}$ state to maintain the $\pi$ orbitals (Figure S10). Conversely, using more m-acceptor ligands (e.g. two $\mathrm{CO}$ ) can enhance the spin delocalization from 
rhenium-center to ligating carbon atoms in the $8 \mathrm{MR}$, leading to an antiaromatic rhenapentalene in the $\mathrm{T}_{1}$ state (Figure $8 \mathrm{c}$ ).

(a)

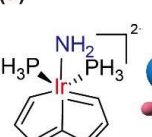
HSOMO

HSOMO-1<smiles>[I-]</smiles>

$(-14.80 \mathrm{eV}) \quad(-16.34 \mathrm{eV})$

(b) HSOMO HSOMO-1

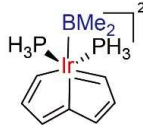

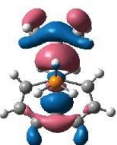

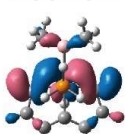

\begin{tabular}{|cccc|}
\hline \multicolumn{2}{c}{ NICS $(1)_{z z}$} & $\pi-E D D B$ & Aromaticity \\
So & -19.3 & $4.41 \mathrm{e}$ & Aromatic \\
$\mathrm{T}_{1}$ & -19.2 & $2.14 \mathrm{e} / / 2.21 \mathrm{e}$ & Aromatic \\
\hline
\end{tabular}

$(-14.16 \mathrm{eV}) \quad(-14.41 \mathrm{eV})$

(c) HSOMO HSOMO-1 (1)
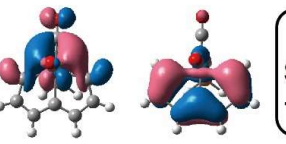

NICS $(1)_{z z} \pi-E D D B \quad$ Aromaticity So $-31.8 \quad 5.26 \mathrm{e} \quad$ Aromatic

$(-10.18 \mathrm{eV}) \quad(-11.10 \mathrm{eV})$

Figure 8. Biorthogonalized SOMOs and aromatic characters of Ir. $\mathrm{NH}_{2}$, Ir. $\mathbf{B M e}$, and $\operatorname{Re} .(\mathrm{CO})_{2}$. Isovalue for MOs: 0.03 a.u. NICS(1)zz values are given in ppm. The $\pi$-EDDB values before and after the "/l" correspond to $\alpha$ and $\beta$ components, respectively.

In summary, using strong donor ligands at the equatorial position is effective in achieving adaptive aromaticity of $16 \mathrm{e}$ metallapentalenes (Figure 9). The more electron-deficient the metal-center $(\mathbf{M})$ is, the stronger donor-ability of the ligand $(\mathbf{L})$ is required. $\pi$-donor and $\sigma$-donor ligands act on $\pi$ and $\sigma$ orbitals, respectively, but both promote the formation of adaptive aromaticity by resulting in the electron delocalization in the $T_{1}$ state comparable to that in the $S_{0}$ state: $\pi-E D D B\left(S_{0}\right) \approx \pi-$ $\operatorname{EDDB}\left(T_{1}\right)$. The ligand effect can be rationalized from two point of views, spin delocalization and electronic transition, which are equivalent in most cases. For species (e.g. Ir.BMe 2 ) whose SOMOs $\left(T_{1}\right)$ do not match the HOMO/LUMO $\left(S_{0}\right)$, the spin delocalization scheme can be used in the rationalization of adaptive aromaticity.

\section{$\mathbf{L}=$ strong $\pi$-donor}

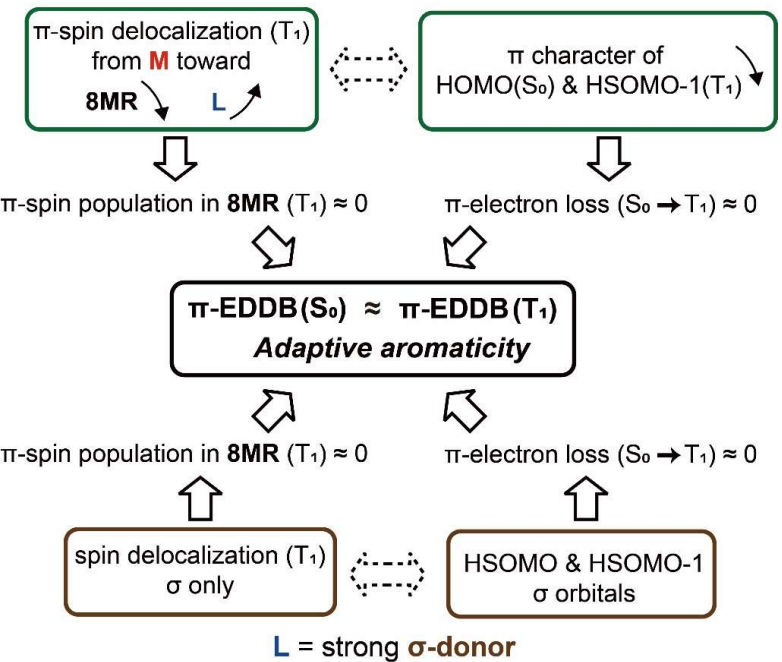

$\sigma$-donor
Figure 9. Strategy for achieving adaptive aromaticity in $16 \mathrm{e}$ metallapentalenes using strong $\pi$ - or $\sigma$-donor ligands.

\section{Conclusion}

Starting from $16 \mathrm{e}$ metallapentalenes with halide ligands, we found unexpected violation of adaptive aromaticity in three iridapentalenes (Ir.F, Ir.CI, and Ir.Br) via NICS, EDDB, and GIMIC approaches. Spin-density distribution and spin-population analyses revealed the distinct spin delocalization accounting for the non-aromaticity of Ir.F, Ir. $\mathbf{C l}$, and Ir. $\mathbf{B r}$ in the $\mathrm{T}_{1}$ state. The stronger $\pi$-donor ability of the iodide is able to prevent spin delocalization in the 8MR of Ir.I $\left(T_{1}\right)$, and thus resulting in the adaptive aromaticity as found in rhenapentalenes and osmapentalenes. A series of ligands with varying $\sigma$ - and $\pi$-donor abilities have been examined, showing that to keep adaptive aromaticity electron-deficient metal-centers are especially demanding of stronger donor ligands. Rhenapentalenes (Re.L) have higher ligand-tolerance with respect to the triplet-state aromaticity. To obtain adaptive aromaticity in osmapentalenes (Os.L), weak donor or even $\pi$-acceptor ligands should not be used. The choice of ligands for iridapentalene (Ir.L) is more limited due to the high electron-deficiency of iridium center. A number of species with adaptive aromaticity have been discovered in this study by using donor-ligands in $16 \mathrm{e}$ metallapentalenes. Our findings not only provide a strategy for achieving adaptive aromaticity in $16 \mathrm{e}$ metallapentalenes but also could inspire the tailoring of more novel aromatics in the excited state.

\section{Computational Methods}

All DFT calculations in this study were performed using the Gaussian 09 software package. ${ }^{[30]}$ Molecular geometries were optimized at the (U)PBE0-D3BJ/6-31G(d) LanL2DZ level[ ${ }^{[31]}$ without symmetry constraints. Open-shell systems were treated with the unrestricted formalism. The 6 $31 \mathrm{G}(\mathrm{d})$ was used for $\mathrm{C}, \mathrm{H}, \mathrm{O}, \mathrm{N}, \mathrm{B}$, and $\mathrm{F}$ atoms while the Lan2LDZ was used for the rest of atoms with $f$ - and $d$-polarization functions for transitionmetal and $p$-block elements, respectively. ${ }^{[32]}$ Spin-orbit coupling was not taken into account. We are aware that hybrid functionals such as the PBEO method overstabilize high-spin states as compared to low-spin states, and therefore, it is likely that the reported singlet-triplet energy gaps $\left(\Delta E_{S T}\right)$ are somewhat underestimated. ${ }^{[3]}$ Frequency calculations were performed to confirm all optimized structures were energy minima. Reliability of geometry optimization at this level has been ensured by testing on an experimentally synthesized osmapentalene (Figure S1). Biorthogonalization of molecular orbitals was carried out using Multiwfn ${ }^{[34]}$ for triplet-state species to obtain one-to-one pairing of $\alpha$ - and $\beta$-spin orbitals, without violating the orthonormalization in each subspace and changing the total energy, total electron density or spin density. Energies of biorthogonalized orbitals were estimated using the Fock matrix obtained

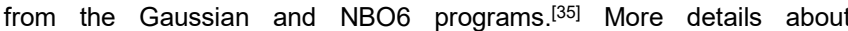
biorthogonalized orbitals can be found in Figure S2. Charge decomposition analyses $(\mathrm{CDA})^{[28]}$ were conducted also using Multiwfn Nucleus-independent chemical shift $(\mathrm{NICS})^{[36]}$ and gauge-including magnetically induced currents (GIMIC) ${ }^{[37]}$ calculations were performed using the gauge invariant atomic orbitals (GIAO). ${ }^{[38]} \mathrm{NICS}(1)_{\mathrm{zz}}$ values were obtained by placing ghost atoms at $1 \AA$ above/below the ring centers at the (U)B3LYP/def2-TZVP//(U)PBE0-D3BJ/6-31G(d) LanL2DZ level of theory (Figure S3). Electron density of delocalized bonds (EDDB) ${ }^{[39]}$ analyses based on density matrices within the natural atomic orbital (NAO) representation were carried out using the RunEDDB script program. ${ }^{[40]}$ 
The long-range corrected functional CAM-B3LYP was used in GIMIC and EDDB calculations for the better description of electron delocalization. ${ }^{[41]}$ The def2-TZVP basis set ${ }^{[42]}$ was used in EDDB calculations for higher accuracy. The complementary results involving the harmonic oscillator model of aromaticity (HOMA $)^{[43]}$ are provided in Table S2 and Figure S4 in the SI but they will not be discussed in detail here.

\section{Acknowledgements}

This work was supported with funds from the China Scholarship Council (CSC) by a State Scholarship Fund (No. 201906310040, D.C.), the National Science Foundation of China (21573179, J.Z.), the Top-Notch Young Talents Program of China (J.Z.), the Ministerio de Economía y Competitividad (MINECO) of Spain (project CTQ2017-85341-P), and the Generalitat de Catalunya (project 2017SGR39). D.W.S. acknowledges the financial support by the European Union's Framework Programme for Research and Innovation Horizon 2020 (2014-2020) under the Marie Skłodowska-Curie Grant Agreement No. 797335 "MulArEffect".

\section{Conflict of interest}

The authors declare no conflict of interest.

Keywords: aromaticity $\cdot$ delocalization $\cdot$ density functional calculations $\bullet$ metallacycles $\bullet$ triplet state 


\section{Entry for the Table of Contents}

Insert graphic for Table of Contents here. ((Please ensure your graphic is in one of following formats))

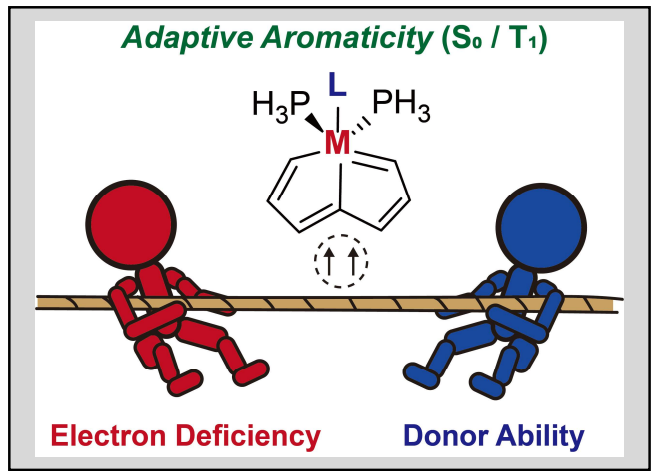

Adaptive aromaticity is a rare property referring to being aromatic in both ground and excited states. The origin of adaptive aromaticity in $16 \mathrm{e}$ metallapentalenes is proved by a spin delocalization mechanism where the donor ability of ligand $(L)$ is competing with the electron deficiency of metal center. Strong $\sigma$ or $\pi$ donor ligands help achieving adaptive aromaticity.

Institute and/or researcher Twitter usernames: @IQCC_UdG @DIMOCAT_iqcc @miquelsola @dszczpnk

\section{References}

[1] a) P. v. R. Schleyer, H. Jiao, Pure Appl. Chem. 1996, 68, 209218; b) P. v. R. Schleyer, Chem. Rev. 2001, 101, 1115-1118.

[2] M. Faraday, Philos. Trans. R. Soc. London 1825, 115, 440-466.

[3] a) E. Hückel, Z. Elektrochemie 1937, 43, 752-788, 827-849; b) N.

C. Baird, J. Am. Chem. Soc. 1972, 94, 4941-4948.

[4] M. Mandado, A. M. Graña, I. Pérez-Juste, J. Chem. Phys. 2008 129,164114

[5] M. Solà, WIREs Comput. Mol. Sci. 2019, 9, e1404.

[6] a) J. R. Bleeke, Chem. Rev. 2001, 101, 1205-1228; b) I. Fernández, G. Frenking, G. Merino, Chem. Soc. Rev. 2015, 44, 6452-6463; c) J. M. Mercero, A. I. Boldyrev, G. Merino, J. M. Ugalde, Chem. Soc. Rev. 2015, 44, 6519-6534; d) F. Feixas, E. Matito, J. Poater, M. Solà, WIRES: Comput. Mol. Sci. 2013, 3, 105-122. [7] a) N. Martin, L. T. Scott, Chem. Soc. Rev. 2015, 44, 6397-6400; b) M. K. Cyrañski, T. M. Krygowski, A. R. Katritzky, P. v. R. Schleyer, J. Org. Chem. 2002, 67, 1333-1338; c) M. Solà, Front. Chem. 2017, 5, 22.

[8] a) H. Ottosson, Nat. Chem. 2012, 4, 969-971; b) M. Rosenberg, C. Dahlstrand, K. Kilså, H. Ottosson, Chem. Rev. 2014, 114, 53795425; c) J. Oh, Y. M. Sung, Y. Hong, D. Kim, Acc. Chem. Res. 2018, $51,1349-1358$.

[9] a) B. Oruganti, J. Wang, B. Durbeej, Org. Lett. 2017, 19, 48184821 ; b) J. Sturala, M. K. Etherington, A. N. Bismillah, H. F. Higginbotham, W. Trewby, J. A. Aguilar, E. H. C. Bromley, A.-J. Avestro, A. P. Monkman, P. R. McGonigal, J. Am. Chem. Soc. 2017, 139, 17882-17889.

[10] a) N. M. Gallagher, A. Olankitwanit, A. Rajca, J. Org. Chem. 2015, 80, 1291-1298; b) M. Mauksch, S. B. Tsogoeva, Phys. Chem. Chem. Phys. 2017, 19, 4688-4694.

[11] S. Shokri, J. Li, M. K. Manna, G. P. Wiederrecht, D. J. Gosztola A. Ugrinov, S. Jockusch, A. Y. Rogachev, A. J.-L. Ayitou, J. Org. Chem. 2017, 82, 10167-10173.

[12] a) O. El Bakouri, J. R. Smith, H. Ottosson, J. Am. Chem. Soc. 2020, 142, 5602-5617; b) N. V. Korovina, C. H. Chang, J. C. Johnson, Nat. Chem. 2020, 12, 391-398; c) L. Wang, L. Lin, J. Yang, Y. Wu, H. Wang, J. Zhu, J. Yao, H. Fu, J. Am. Chem. Soc. 2020 doi:10.1021/jacs.1020c00089.
[13] a) D. Chen, T. Shen, K. An, J. Zhu, Commun. Chem. 2018, 1 , 18; b) T. Shen, D. Chen, L. Lin, J. Zhu, J. Am. Chem. Soc. 2019, $141,5720-5727$

[14] a) D. P. Craig, N. L. Paddock, Nature 1958, 181, 1052-1053; b) M. Mauksch, S. B. Tsogoeva, Chemphyschem 2016, 17, 963-966. [15] D. Chen, Q. Xie, J. Zhu, Acc. Chem. Res. 2019, 52, 1449-1460. [16] D. Chen, R. Qiu, S. Dong, J. Zhu, Theor. Chem. Acc. 2020, 139 121.

[17] a) C. Zhu, S. Li, M. Luo, X. Zhou, Y. Niu, M. Lin, J. Zhu, Z. Cao, X. Lu, T. Wen, Z. Xie, P. V. Schleyer, H. Xia, Nat. Chem. 2013, 5, 698-703; b) C. Zhu, M. Luo, Q. Zhu, J. Zhu, P. v. R. Schleyer, J. I. $\mathrm{Wu}, \mathrm{X}$. Lu, H. Xia, Nat. Commun. 2014, 5, 3265.

[18] a) E. Hückel, Z. Phys. 1931, 70, 204-286; b) E. Heilbronner, Tetrahedron Lett. 1964, 5, 1923-1928.

[19] a) R. Grande-Aztatzi, J. M. Mercero, E. Matito, G. Frenking, J. M. Ugalde, Phys. Chem. Chem. Phys. 2017, 19, 9669-9675; b) D. W. Szczepanik, M. Solà, ChemistryOpen 2019, 8, 219-227.

[20] M. Baranac-Stojanović, J. Org. Chem. 2020, 85, 4289-4297.

[21] E. Ruiz, J. Cirera, S. Alvarez, Coord. Chem. Rev. 2005, 249, 2649-2660.

[22] H. Fallah-Bagher-Shaidaei, C. S. Wannere, C. Corminboeuf, R. Puchta, P. v. R. Schleyer, Org. Lett. 2006, 8, 863-866.

[23] a) R. Herges, D. Geuenich, J. Phys. Chem. A 2001, 105, 32143220; b) D. Geuenich, K. Hess, F. Köhler, R. Herges, Chem. Rev. 2005, 105, 3758-3772.

[24] C. Foroutan-Nejad, J. Vícha, A. Ghosh, Phys. Chem. Chem. Phys. 2020, 22, 10863-10869.

[25] D. W. Szczepanik, M. Andrzejak, J. Dominikowska, B. Pawelek, T. M. Krygowski, H. Szatylowicz, M. Solà, Phys. Chem. Chem. Phys. 2017, 19, 28970-28981.

[26] D. W. Szczepanik, Comput. Theor. Chem. 2017, 1100, 13-17. [27] G. Frenking, S. Fau, C. M. Marchand, H. Grützmacher, J. Am. Chem. Soc. 1997, 119, 6648-6655.

[28] S. Dapprich, G. Frenking, J. Phys. Chem. 1995, 99, 9352-9362. [29] a) A. Dachs, A. Torrent, A. Roglans, T. Parella, S. Osuna, M. Solà, Chem. -Eur. J. 2009, 15, 5289-5300; b) W. Zheng, A. Ariafard, Z. Lin, Organometallics 2008, 27, 246-253; c) S. A. Macgregor, D. C Roe, W. J. Marshall, K. M. Bloch, V. I. Bakhmutov, V. V. Grushin, J. Am. Chem. Soc. 2005, 127, 15304-15321. 
[30] M. J. Frisch, G. W. Trucks, H. B. Schlegel, G. E. Scuseria, M. A Robb, J. R. Cheeseman, G. Scalmani, V. Barone, B. Mennucci, G. A. Petersson, H. Nakatsuji, M. Caricato, X. Li, H. P. Hratchian, A. F. Izmaylov, J. Bloino, G. Zheng, J. L. Sonnenberg, M. Hada, M. Ehara, K. Toyota, R. Fukuda, J. Hasegawa, M. Ishida, T. Nakajima, Y. Honda, O. Kitao, H. Nakai, T. Vreven, J. A. Montgomery, J. E. Peralta, F. Ogliaro, M. Bearpark, J. J. Heyd, E. Brothers, K. N. Kudin, V. N. Staroverov, R. Kobayashi, J. Normand, K.

Raghavachari, A. Rendell, J. C. Burant, S. S. Iyengar, J. Tomasi, M. Cossi, N. Rega, J. M. Millam, M. Klene, J. E. Knox, J. B. Cross, V. Bakken, C. Adamo, J. Jaramillo, R. Gomperts, R. E. Stratmann, O. Yazyev, A. J. Austin, R. Cammi, C. Pomelli, J. W. Ochterski, R. L. Martin, K. Morokuma, V. G. Zakrzewski, G. A. Voth, P. Salvador, J. J. Dannenberg, S. Dapprich, A. D. Daniels, Ö. Farkas, J. B. Foresman, J. V. Ortiz, J. Cioslowski, D. J. Fox, Gaussian 09, Revision E.01, Gaussian, Inc., Wallingford CT, 2013.

[31] a) J. D. Chai, M. Head-Gordon, J. Chem. Phys. 2008, 128, 084106; b) S. Grimme, S. Ehrlich, L. Goerigk, J. Comput. Chem. 2011, 32, 1456-1465; c) W. J. Hehre, R. Ditchfield, J. A. Pople, J. Chem. Phys. 1972, 56, 2257-2261; d) P. C. Hariharan, J. A. Pople, Theoret. Chim. Acta 1973, 28, 213-222; e) P. J. Hay, W. R. Wadt, J. Chem. Phys. 1985, 82, 299-310.

[32] a) A. W. Ehlers, M. Böhme, S. Dapprich, A. Gobbi, A. Höllwarth, V. Jonas, K. F. Köhler, R. Stegmann, A. Veldkamp, G. Frenking, Chem. Phys. Lett. 1993, 208, 111-114; b) C. E. Check, T. O. Faust, J. M. Bailey, B. J. Wright, T. M. Gilbert, L. S. Sunderlin, J. Phys. Chem. A 2001, 105, 8111-8116.

[33] a) M. Swart, J. Chem. Theory Comput. 2008, 4, 2057-2066; b) M. Reiher, O. Salomon, B. Artur Hess, Theor. Chem. Acc. 2001, 107, 48-55; c) M. Güell, M. Solà, M. Swart, Polyhedron 2010, 29, 8493.
[34] T. Lu, F. Chen, J. Comput. Chem. 2012, 33, 580-592.

[35] E. D. Glendening, J. K. Badenhoop, A. E. Reed, J. E. Carpenter J. A. Bohmann, C. M. Morales, C. R. Landis, F. Weinhold, NBO 6.0,

Theoretical Chemistry Institute, University of Wisconsin, Madison, 2013.

[36] a) P. v. R. Schleyer, C. Maerker, A. Dransfeld, H. Jiao, N. J. R. v. E. Hommes, J. Am. Chem. Soc. 1996, 118, 6317-6318; b) Z. Chen, C. S. Wannere, C. Corminboeuf, R. Puchta, P. v. R. Schleyer, Chem. Rev. 2005, 105, 3842-3888.

[37] a) J. Jusélius, D. Sundholm, J. Gauss, J. Chem. Phys. 2004, 121, 3952-3963; b) H. Fliegl, S. Taubert, O. Lehtonen, D. Sundholm Phys. Chem. Chem. Phys. 2011, 13, 20500-20518; c) D. Sundholm, H. Fliegl, R. J. F. Berger, WIREs Comput. Mol. Sci. 2016, 6, 639678.

[38] K. Friedrich, G. Seifert, G. Großmann, Z. Phys. D -Atom. Mol. Cl. 1990, 17, 45-46.

[39] D. W. Szczepanik, M. Andrzejak, K. Dyduch, E. Zak, M. Makowski, G. Mazur, J. Mrozek, Phys. Chem. Chem. Phys. 2014, 16, 20514-20523.

[40] D. W. Szczepanik, RunEDDB, available at: http://eddb.pl/runeddb/ (accessed in February 2020).

[41] a) T. Yanai, D. P. Tew, N. C. Handy, Chem. Phys. Lett. 2004, 393, 51-57; b) D. W. Szczepanik, M. Solà, M. Andrzejak, B.

Pawelek, J. Dominikowska, M. Kukulka, K. Dyduch, T. M. Krygowski, H. Szatylowicz, J. Comput. Chem. 2017, 38, 1640-1654.

[42] F. Weigend, R. Ahlrichs, Phys. Chem. Chem. Phys. 2005, 7 , 3297-3305

[43] T. M. Krygowski, J. Chem. Inf. Comput. Sci. 1993, 33, 70-78. 